\title{
Dark-field hyperlens exploiting a planar fan of tips
}

\author{
Henri Benisty* and François Goudail \\ Laboratoire Charles Fabry, Institut d'Optique, CNRS, Univ P Sud, 2 Avenue Augustin Fresnel, Palaiseau, France \\ ${ }^{*}$ Corresponding author: henri.benisty@institutoptique.fr
}

Received June 19, 2012; revised July 19, 2012; accepted July 27, 2012;

posted July 30, 2012 (Doc. ID 170914); published August 30, 2012

\begin{abstract}
Metallo-dielectric superlenses transfer subwavelength-scale information without magnification. The so-called hyperlenses additionally magnify, transferring images into traditional far-field optics. We target hyperlenses based on the "canalization" phenomenon in an array of wires, modified to form an open fan, also called "endoscope." We use an integrated optics design with silicon wires, fed for instance by grating couplers, accessing gold wire fans. This alleviates the need to care for wire length. We explore a regime where we do not only image a near-field source, but where we image illuminated nano-objects, as done in microscopy, light being fed by a second fan before the object plane. In order to counter the low contrast from illuminated nano-objects, we propose here a dark-field hyperlens concept: We show that the illumination fan can be fed so as to get a dark output for a "void" object field, as occurs in the eponym microscopy method. We obtain, at a wavelength as large as $1200 \mathrm{~nm}$, a well-resolved imaging capability for a scene of two $30 \mathrm{~nm}$ silicon particles. (c) 2012 Optical Society of America OCIS codes: $\quad 180.4243,100.6640,250.5403,180.3170$.
\end{abstract}

\section{INTRODUCTION}

The extension of the concepts of optical/spatial information transfer to the subwavelength scale (SWS) has been boosted in recent years by innumerous near-field techniques, contributing to nano-optics [1] . Superlensing phenomena made popular after Pendry's proposal [2], showed that longitudinal SWS information transfer was possible with metallo-dielectric (MD) structures, even though the field exponential growth/ decay and the losses involved some limits [3]. It then became logical to introduce the equivalent of traditional microscopy imaging in the form of a transfer of several parallel information in an object plane from SWS to superwavelength scale, hence the hyperlens. Unlike the many single-tip-based techniques such as scanning-near-field optical microscopy [SNOM, Fig. 1(a)] that only achieved that job for a single "pixel" at a time at the tip apex, a hyperlens may transfer information simultaneously from an "object field" that comprises many degrees of freedom, addressing them to many "pixels" at the camera end of a typical setup. Following the exploration of metamaterials, the two main flavors of hyperlenses to date [Fig. 1(b)] have been based on either the "canalization" phenomena from an array of metallic wires (see below) or on curved MD stacks [4]. In the latter, the hyperbolic dispersion of MD flat superlattices can be used to address different wavevectors well in SWS regime as a function of frequency.

In the case of "canalization," whose operation is more obvious, wires or tips carry free charges and transfer SWS fields along their surface [ $\underline{5}-\underline{19}$ ], with a resolution limited by skindepth. There have been several studies to assess the degree of information transfer without mixing, and notably the perturbation caused by the change of impedance at the wire ends, making their length crucial in the abrupt-cut conditions. One simple reason is that it behaves as a very particular FabryPerot system, given the special dispersion that turns out to be invariant for any of its spatial harmonics [20]. This makes it possible to also envision imaging inside such wire arrays [20,21], an attractive option for $\mathrm{THz}$ or microwave wavelengths, providing also physical inspiration for hyperlenses in general. Comparatively few studies $[\underline{9}, \underline{10}, \underline{12}]$ have addressed the magnification regime, but its main ingredients are similar, and it is obviously most critical on the object side. The term of endoscope [22] was coined for such "fans" of wires. Related realizations were studied in the acoustical regime [23]. It is clear that the relative growth of the skin depth to wavelength ratio in the optical regime makes it more difficult to avoid mixing across wires at shorter wavelengths. But the recent trends of metamaterials toward optical frequency operation, making the best use of the role of surface plasmons (SP) [24,25], suggests that well-designed systems may acceptably perform.

Demonstrations to date have emphasized the hyperlensing magnification of SWS sources, or of objects with a relatively large cross-section such as full metal lines of $35 \mathrm{~nm}$ in the pioneer case $[\underline{4}, 26]$. However, the process of imaging illuminated nano-objects and reading out the SWS information, in a manner truly parallel to standard microscopy, has not been satisfactorily achieved. One simple reason is that the cross section of such SWS objects is small.

The largely historical attempts to image faintly scattering objects have given rise to "dark-field" techniques (see [27] for instance), [Fig. 1(c)].

The oldest ones, still much in use, amount to illuminate only at large transverse wavectors $k_{\perp}$, say $k_{\perp}>\mathrm{NA} k_{o}\left(k_{o}=2 \pi / \lambda\right.$ vacuum wavevector, NA numerical aperture), and discard direct (or specular) rays, collecting only rays at $k_{\perp}<\mathrm{NA} k_{o}$. Among the recent notable successful attempts, [28] has proposed the use of a Michelson interferometer, which amounts to a homodyne scheme whereby a reflected wave is read with a signal-to-noise ratio scaling like its amplitude reflectivity $r$ and not as the $|r|^{2}$ scaling for standard direct detection. Quick and sensitive detection of virus-size particles was demonstrated. Infrared mapping of viruses was also done [29], but with a tip having a $\sim 15 \mathrm{~nm}$ apex, hence slowly.

To further add SWS resolution to any such scheme, we have had in mind the remarks by M. Fink and co-workers [30], 

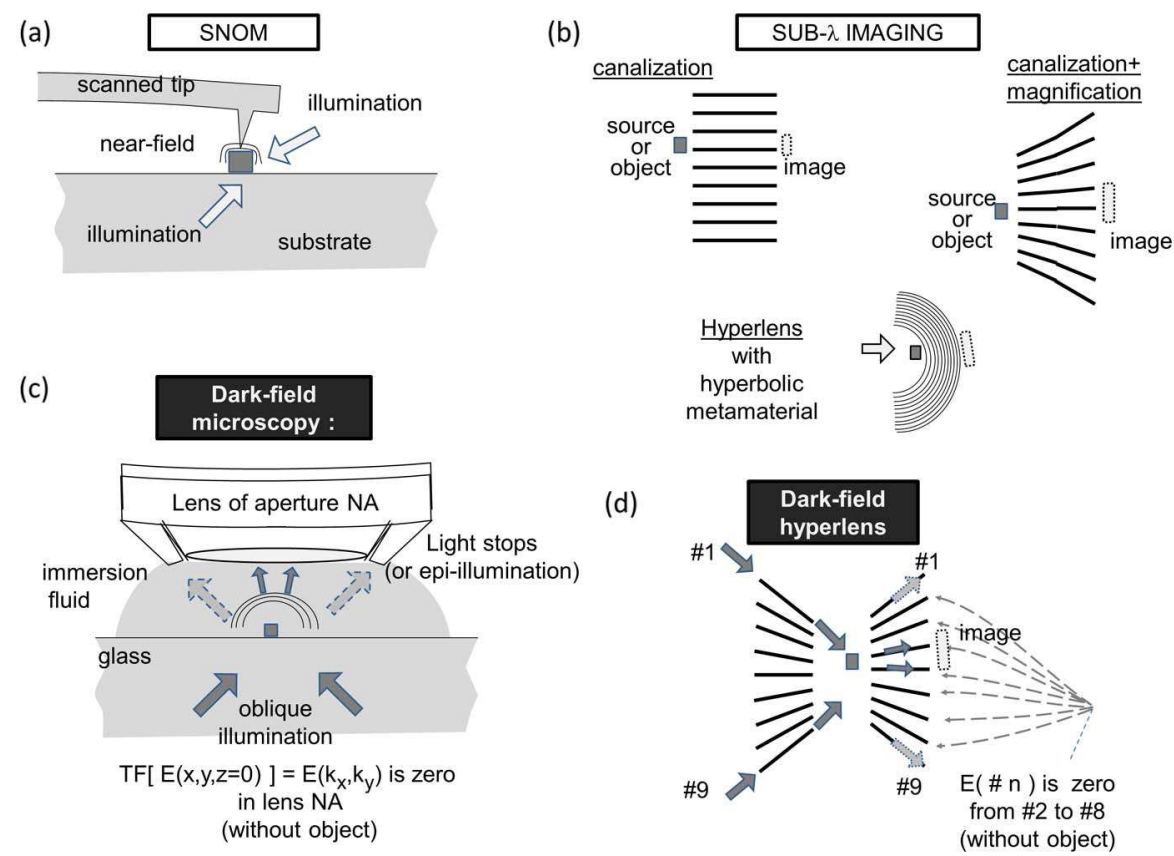

Fig. 1. (Color online) Common and proposed microscopy principles: (a) SNOM scans a subwavelength-sized tip on the objects; (b) subwavelength imaging makes use of canalization along metal wires without or with magnification (with a fan of wires), or of rolled hyperbolic materials; (c) dark-field microscopy uses oblique illumination not detected in direct path, and captures scattered light only; (d) the proposed dark-field hyperlens aims at implementing the same principle between two fans of wires.

prompted by their studies on time-reversal imaging in acoustics, implying that the usual resolution limit (Rayleigh criterion) can be ascribed to the smooth shape of the Green function in vacuum/uniform medium (the "background" of the objects to be imaged), and that consequently, a SWS structured Green function is needed to beat it. There are indeed several structured illumination/structured medium schemes currently reported, among which we can cite grating-induced high resolution imaging proposals [31], intimately and cleverly structured anisotropic media [32,33], or use of localized vibrating motion for a similar purpose [34].

In our case, the structure comes from the simple juxtaposition of several tips in the vicinity of the imaging field $(2 \times 7$ metallic tips here, addressed through $2 \times 6$ silicon wires).

Even though the Green function of such an ensemble $G_{F}\left(x, x^{\prime}, \omega\right)$ between a point $x$ of the object field and a point $x^{\prime}$ of the exit ( $\sim$ image field) can allow SWS imaging, it will in general not yield a big signal change between a void object field and an object field with a SWS particle in it, because the image is already "bright." While in usual optics, dark-field is nearly as easily done in reflection and in transmission, we believe it is difficult to cancel reflection at all in the case of generic MD structures. Therefore, we focus on transmission dark-field, attempting to cancel the output signals in the absence of particle. This defines the "dark-field hyperlens" concept sketched in Fig. 1(d), and that is detailed below. Given the dark background, we get a large relative signal even for a single dielectric particle in the object field with index ratio over background of less than 2 .

In other words, in usual dark-field imaging, the input field is arranged so as to give negligible light in the detecting channel through the device, usually by a simple mean such as annular lighting, attempting conversely to collect light scattered in the field of the lens for detection. In general terms, it is a restriction among the possible waves impinging on the device, further broken by the scattering particle. We attempt to have the same result of negligible light in the detecting channel and broken restriction by a particle, but the price we shall pay to reach the subwavelength regime will be a more complex input field arrangement, and a more demanding work to interpret the scattered light pattern back into the position of single or multiple particles in the field.

\section{DESCRIPTION OF THE DEVICE}

We now turn to our specific proposal of a transmission darkfield hyperlens (TDFH). We address the object plane with two facing fans of metallic tips in the spirit of the works by Belov's team [5-10,12-19], also termed endoscope [22] and sketched in planar form in Figs. 1(c) and 1(d).

We assume such tips to be laid over a silica substrate, as shown in Fig. 2(a), and to be eventually embedded in silica, so our background index is $n_{B}=1.5$. A similar index $n_{B} \sim$ $1.35-4$ would be a plausible situation if we were sensing a fluidic channel for instance, immobilizing particles of interest in the object plane. To "optically contact" these metallic tips in the $x y$ plane, we could use specific plasmonic couplers from a top direction (z), analogous to those popular for SoI chips [35-37]. We prefer an arrangement where we first feed larger silicon wires with such well known grating coupling solutions and next couple these $\mathrm{Si}$ wires to the metallic tips.

Thus, in the rest of this work we simplified the situation to a 2D $x y$ plane. We believe that this does not affect the operation principle that we are going to detail. For instance, we will need phase control of the individual tips, which would be easily implemented with silicon wires and existing modulating solutions developed for instance for heterodyne detection in silicon photonics [38-41]. Exits are similarly described here in 2D. But they could be arranged to get light outcoupling directly under a microscope so that some equivalent image 
of the particles inside the working area between the two fans would be readily available to the user, a direct image for quick access, producing later a more precise treated image according to the principles detailed below.

To transit between a signal in a $\mathrm{Si}$ wire and a signal in a gold wire that can address the SWS, we make use of the staggered fans depicted in Figs. 2(b) and 2(c) below. They are based on the work by Han et al. [42] whereby the strong field of the TE polarization surrounding the sides of the tip naturally becomes the field corresponding to the surface charges between the two subsequent metallic surfaces. Little reflection occurs in these conditions.

The silicon tips can then be tapered [Fig. 2(c)]. The limit to confinement and resolution essentially comes from the skin depth of the metal [6], on the order of 15-20 nm in the nearinfrared range. The metal tips cannot be shrunk much below without compromising the SWS local concentration of field needed to achieve a "landscaped" Green function and in turn a SWS imaging (SWSi) capability.

As for the gap between the two facing fans, we have to leave less than a $\sim 50 \mathrm{~nm}$ gap to be sure that the working plane of the system (the device central axis $x=0$ ), is able to couple local scale information to the fan of tips. This departs from a ray-tracing intuition whereby the two fans' centers would be collocated at some central focus.

Another aspect of the range investigated here is that it allows adjacent tips to mix their information along the propagation path, not only the metal tips $[\underline{8}, \underline{9}, \underline{12}, \underline{13}, \underline{16}]$ but also the silicon tips because we position them as close as possible from the objects to minimize losses. This is very analogous

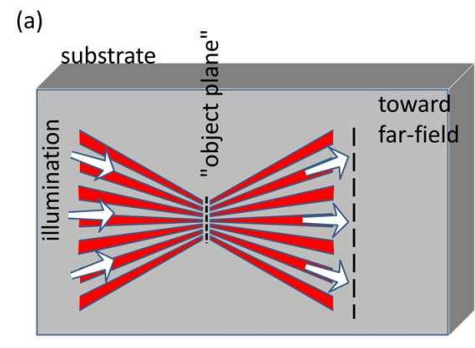

(c)
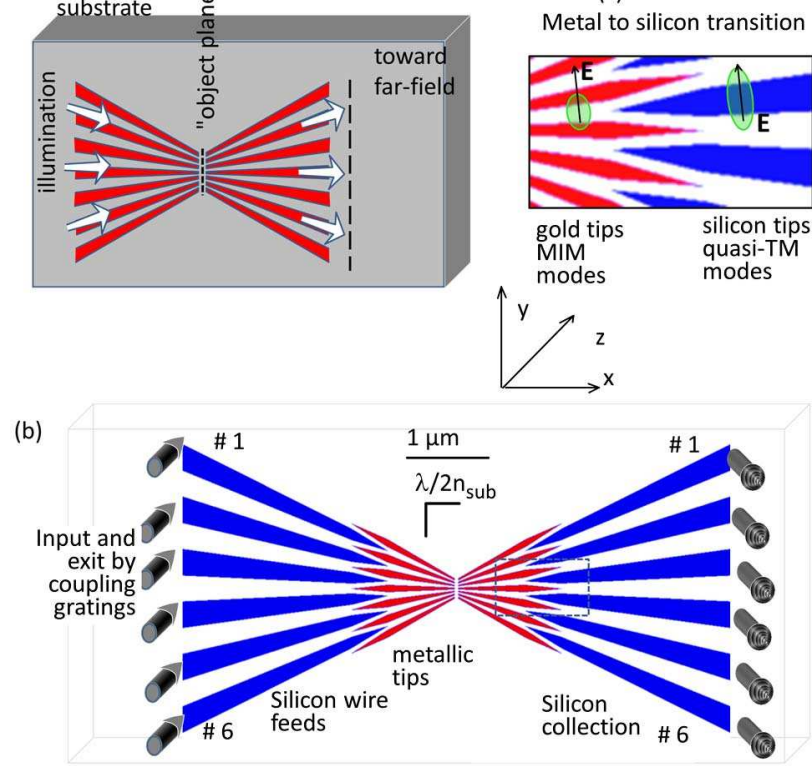

Fig. 2. (Color online) (a) Fan of metallic tips addressing a central "object plane," where particle detection is desired, at subwavelength scale. (b) The fan of seven tips can be addressed through six silicon wires 1...6. Light in these wires can further be in- or out-coupled in the far-field and the outside world through gratings on them. (c) Magnification of the dashed rectangle area indicated in (b): transition between the metal and silicon parts. The field is confined between the metal tips (the so-called MIM configuration) and this information is further transferred to the quasi-TM modes of the silicon wires, whose field is also prominent outside them. to what has been documented at the "star-side" of waveguides arrays in well-documented phasar/AWGs devices [43]. Given this state of matter, we will call in brief "tips" also the silicon photonic wires, even though each of them topologically addresses two metallic tips: the extra photonic mixing complexifies the situation, so that, as far as imaging is concerned, we will eventually have to revisit the concept of a point spread function in our specific context. We will see however that with the help of dark field, SWS information can still be obtained.

\section{ENGINEERING THE DEVICE'S GREEN FUNCTION}

To study this system, we now launch fields in each silicon tip (\#1 to \#6) in the form of the local calculated TE profile for a silicon waveguide of constant width and $x$ axis. We use only a 2D finite-difference time-domain (FDTD) method without dispersion and a single polarization, by using a corresponding effective index that accounts for the vertical confinement. The silicon index then becomes 2.8, typical of silicon photonics with silicon thicknesses of $\sim 220 \mathrm{~nm}$, while the metal index is taken as a Drude metal with $\mathrm{Au}$ characteristics from Johnson and Christy [44] fitted at vacuum wavelength $\lambda=1200 \mathrm{~nm}$. The FDTD grid was taken quite small (down to $3.0 \mathrm{~nm}$ ). Nevertheless, the discretization of oblique walls in FDTD is well known to produce hot spots. We will discuss below the role of these "constant imperfections" in the simulation. The role of irregularities in superlensing phenomena is being studied with interest [45] as it could be partly positive.

As a first step, when we launch as an excitation a guided field in the $i$ th tip ( $i=1$ to 6 ) on the left of Fig. 2(b), we collect light at all six receivers situated at the end of the silicon tips \#1 to \#6, which altogether collect most of the light propagating to the right side of our device. This collection is also quantified as a projection on the local guide mode. The complex field amplitude retrieved by such a procedure at the $j$ th output (the local complex amplitude of the guided mode) can be written $E(i, j, \omega)$.

Then, by analogy with genuine continuous Green functions, we can say that $E(i, j, \omega)$ represents a discrete version $G_{F}(i, j, \omega)$ that we can still call the tip-to-tip Green function of our ensemble [Fig. 2(b)] between any two tips $\operatorname{Tip}_{i}$ on the entrance, left side, and $\operatorname{Tip}_{j}$ on the exit, right side.

We are now equipped to implement the dark-field operation principle: We collect the information at the exit of only the four central tips $2 \ldots 5$ and let the two extreme ones "dissipate" illumination as in Fig. 1(d). In a sense, we remain with 4 degrees of freedom. Four "pixels" are few when it comes to imaging, but it makes our structure distinctly different from the SNOM-type single-tip SWSi generally requiring a scan of the tip. We then target the cancellation of the exit field in the absence of objects in the field, by properly choosing the complex amplitude of the local waveguide mode sources. At the present stage, we demand this cancellation only at one particular frequency $\omega_{\text {dark }}$, but this is limited only by the ability to control phase and amplitude of each frequency with standard spectral resolution. Because there are six input tips and four exit tips, we may set two of these amplitudes $E_{j}$ to unity or any similar constraint, and determine the four other complex amplitudes such that the four exit ones are cancelled, making for our SWS dark-field hyperlens. 
In an ideally symmetrical layout, there would also be strong symmetries of the source amplitudes resulting from this procedure. However, due to the buildup of hot spots along the meshed MD interfaces combined with the FDTD mesh asymmetry (which we did not control), and the sensitivity of narrow gaps of MD structures in general, a substantial absence of symmetry can be observed, for instance in the Green function $G_{F}(i, j, \omega)$. This issue could be thought to affect the realization of a dark-field, but we can still have an acceptable cancellation and confirm that the principle is quite general. We even believe that it is useful to deal with such substantial imperfections, because they would also occur in practice. This means, for the time being, that the Green function would be devicedependent and that the dark-field condition would have to be trimmed individually in each device, solving a linear signal processing problem of known nature. In our case, based on an examination of the Green function, we actually imposed power on tip \#3 (of 6) as unity, we next forced tips \#2 and \#5 to have opposite amplitudes following the general idea of cancellation in the center of the device (similar to Zernike's strioscopy in far-field imaging), and we then adjusted the remaining four complex amplitudes by standard linear algebra.

In practice, this could be accomplished by feeding the six input waveguides from a single on-chip laser source, followed by $1 \times 6$ splitters, variable optical attenuators and electrooptical phase shifters. Such integrated building-blocks are commonly present in currently developed photonic integrated circuits (PICs) for telecom applications in order to develop a complex phase-amplitude modulation format [ㅎ6].

The result can be seen in Fig. 3(b) in the form of a color map of the outgoing flux in the six exit tips as a function of frequency in the spirit of spectro-imaging. The two side tips are also shown. They carry a lot of field, just as a dark-field lens would in its outer ring. It is seen that at the wavelength of $\lambda_{\text {dark }}=1200 \mathrm{~nm}$ the power is indeed zeroed in the "dark field" compared to all nearby wavelengths (wavenumber $\sigma_{\text {dark }}=8333 \mathrm{~cm}^{-1}$, we use wavenumbers since the Fouriertransformed FDTD output is linear in frequency). Being in principle a linear algebra problem, we should get a perfect cancellation. However, remember that the simulations are made in the time domain, and that small errors are then combined in a not so trivial fashion. As a result, we observe that the linearity of the FDTD is slightly imperfect in the frequency domain, but that it is still sufficient here to get $\sim 1: 100$ to $1: 10$ cancellations for the four tips: the worst case is tip \#5, which, being situated close to the brightest tip \#6, probably gets some influence from the nonlinearity of numerical origin. We did not attempt to fine-tune further the sources since in practice, such imperfect cancellation could also arise. (We remark about these cancellation effects that operation with a feedback loop insuring such a cancellation in the presence of objects would probably retrieve an even better information than the dark-field readout detailed here, but it is more complex to implement yet). The spectral range where cancellation occurs is of about $1-2 \%$ width, say $15-20 \mathrm{~nm}$, suggesting that control over a broader band would be practically feasible. This width is related to the phase sensitivity along the few microns total path, a path which is long enough to produce such variation for modest number of round trips.

There are also substantial losses in our non-optimized design. The power flux in the two extreme bright tips \#1 and \#6 is
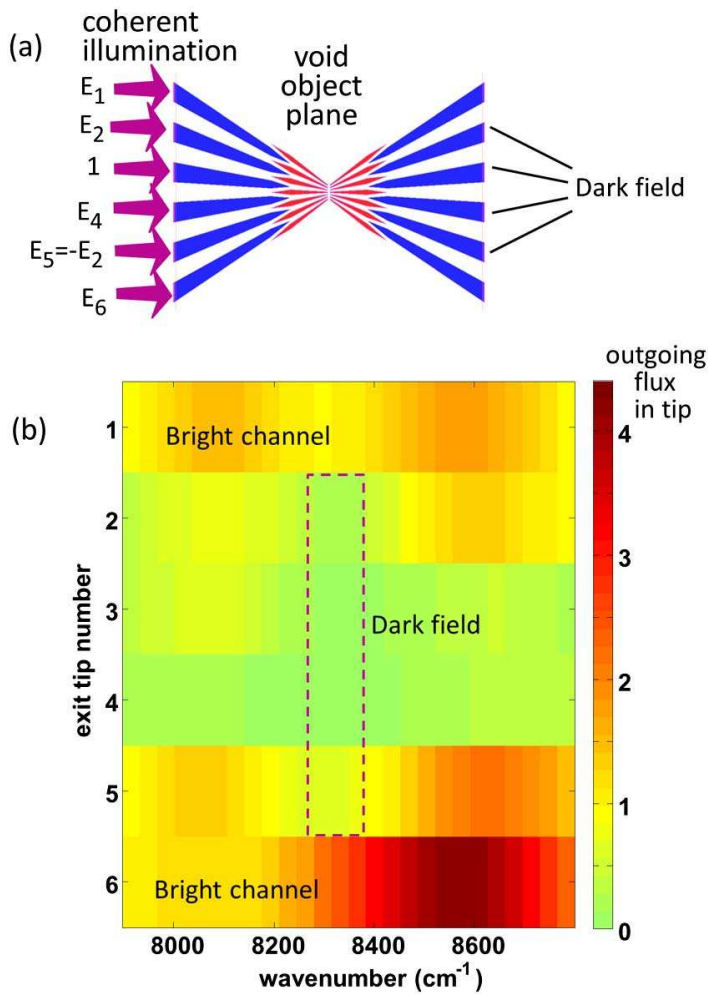

Fig. 3. (Color online) (a) Principle of the dark field configuration: the illumination is coherent in the six wires. In an ideal structure it would be antisymmetrical. In an imperfect structure, the field phases and amplitude are adjusted so as to minimize the field of the four central exit channels, not constraining light in the two outer tips. (b) Spectroimaging color map of output fluxes versus wavenumber. If fields are adjusted for a single wavelength (here $\lambda=1200 \mathrm{~nm}$ ), the field remains "dark" in a spectral region of 1-2\% width. Minimization is seen to be imperfect for tip \#5 on account of its proximity with the brightest channel.

about $10^{-2}(-20 \mathrm{~dB})$ times the power launched at the entrance. It is somewhat ambiguous to define transmission of such a device, however, as the conditions imposed may well have caused reflection. If we attribute one half of this number to each of the traversed fan, the resulting $-10 \mathrm{~dB}$ transmission per fan is not very surprising for a hyperlens in this wavelength range. Our guess from case studies is that some $4-8 \mathrm{~dB}$ may stem from the metal losses, while the rest plausibly comes from imperfect coupling from silicon to metal and of the "scattering" nature of the central zone, partly radiating outside the exit fan.

\section{IMAGING AND SIGNAL PROCESSING}

Our purpose is now to use the signal detected at the output tips in order to estimate the location of nanoparticles in the active region of the device.

In practice, we have to collect optical information in the form of the complex amplitude at the six silicon waveguides. This could be done after outcoupling to air or fibers, but, as we shall see that correlation processing is welcome, collection could be made on the chip, by using the same kind of telecom PICs building-blocks (for amplitude and phase control) that were indicated to fabricate the input signal of interest [46]. Then the correlation would essentially amount to detecting the beat note of each collected signal with a signal derived 
from the original laser with the adequate phase and amplitude. These phase and amplitudes would be those detected for a single particle travelling in the "object plane," as we study in the following paragraph.

Let us first consider a single silicon particle of $30 \mathrm{~nm}$ (square shape, $n=2.8$ ) that is moved through 43 positions from $y=-110 \mathrm{~nm}$ to $y=+100 \mathrm{~nm}$ by $\Delta y=5 \mathrm{~nm}$ steps, as depicted in Fig. 4(a).

As hinted above, such a particle could be a nonfluorescent label in a fluidic channel for on-chip biomolecular recognition experiments. Such a particle moves in a constrained way along the channel axis, and we want to track one or several such particles with subwavelength accuracy. The output signal of the six tips for the 43 considered particle positions is represented on Fig. 5. The expected ideal "geometric" image of the particle, i.e., the image for a perfect canalization scheme, would lie on the black dashed oblique line. Therefore, we see that there is no good "point spread function" for this system, since the signal is very variably located with respect to the geometric image. Nevertheless, we can reasonably guess that the particle position information is acceptably preserved, be it spread among the four complex numbers depicting the dark-field output from tips \#2 to \#5. How unambiguously a single particle position can be retrieved by inversion depends on how well the problem is "conditioned." We do not go into these signal processing aspects here, even though they would be eventually needed to assess the ultimate resolution. Let us work out nevertheless a first step in the form of single-particle correlation function as an extended version of such correlation will be relevant to the rest of our demonstration.

Let us assume that we observe an output signal vector $\vec{E}=$ $\left(E_{2}, \ldots, E_{5}\right)^{T}$ at the four "dark field" tips (the superscript denotes vector transposition). A basic correlation procedure for estimating the position of a single randomly situated particle from this data is to compare it to the reference data $\vec{E}\left(y_{j}\right)$
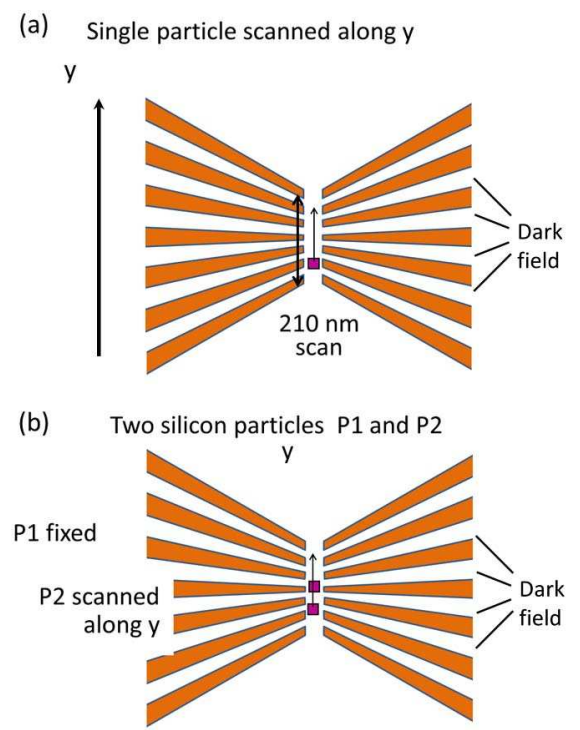

Fig. 4. (Color online) (a) Reference configuration: a single particle is scanned through the "object plane," with a $5 \mathrm{~nm}$ step, yielding $N_{1}$ complex "images" that are read at the 4 exit channels. (b) Situation used to probe hyperlens dark-field imaging: one particle P1 moving as in the reference case, the other particle $\mathrm{P} 2$ immobilized at $5 \mathrm{~nm}$ above the $y=0$ axis.

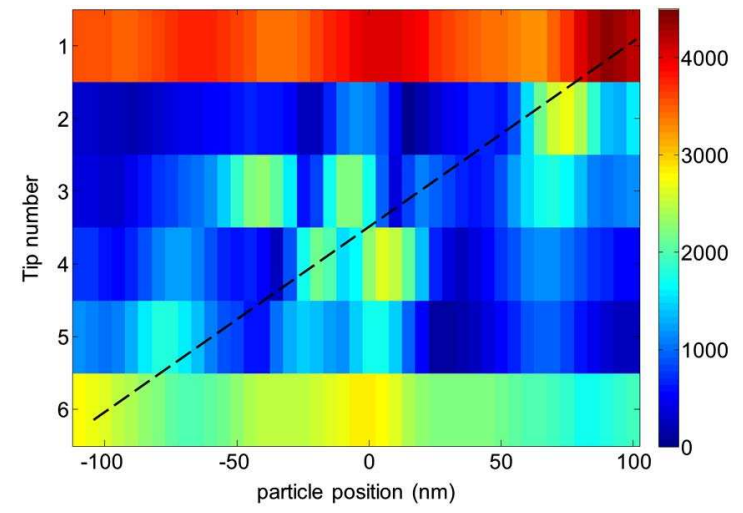

Fig. 5. (Color online) Hyperlens field modulus $|E|$ at the 6 exit tips at $\lambda_{\text {dark }}$ in a color map for a single Si particle, as a function of particle position. The dashed tilted line represents the expected "geometric" image position, going linearly from one extreme to the other extreme tip of the field.

where $y_{j}$ represents one of the 43 positions in Fig. 5 . A possible way of making this comparison is to compute the scalar products $\vec{E}+\vec{E}\left(y_{j}\right)$, and select the position estimate $\hat{y}$ that maximizes this product:

$$
\hat{y}=\operatorname{argmax}_{j}\left[\vec{E}^{+} \vec{E}\left(y_{j}\right)\right],
$$

where the superscript + denotes transconjugation. Normalization of single-particle data simply reads $\vec{E}\left(y_{j}\right)+\vec{E}\left(y_{j}\right)=1$. To validate the feasibility of this technique, we have represented in Fig. 6 the cross scalar product between the signals $\vec{E}(y)$ for each pair of positions $\left(y_{j 1}, y_{j 2}\right)$ :

$$
A\left(y_{j 1}, y_{j 2}\right)=\vec{E}^{+}\left(y_{j 1}\right) \vec{E}\left(y_{j 2}\right)
$$

We observe that this function decreases rapidly away from the diagonal, showing the relevance of this correlation to estimate the position of the particle. We can observe some local maxima in nondiagonal positions that could lead to estimation ambiguities. They can be overcome in practice if the detection scheme has a sufficient signal-to-noise ratio, or by enforcing trajectory consistency when a particle is being tracked.

However, this alone does not prove SWS imaging because if we have two particles in the object field, we have in this

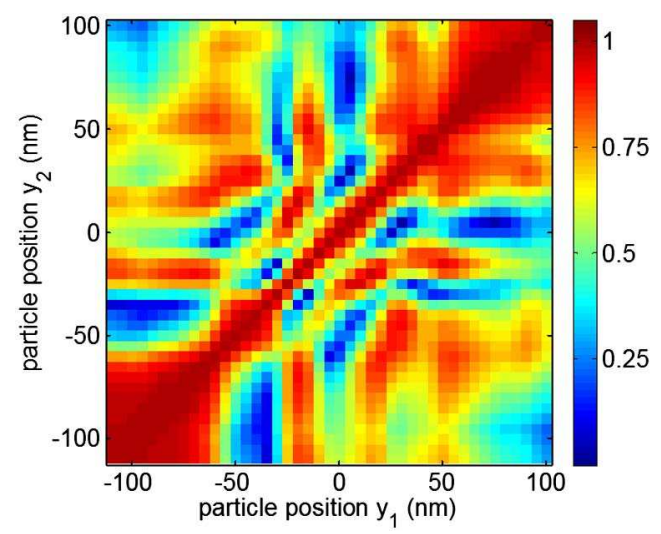

Fig. 6. (Color online) Self correlation of single-particle fields, peaking at unity on the diagonal. 
particular hyperlens as in many other ones, no insurance that the scattered fields by a given particle do not again interact with other particles being imaged, because of the highly scattering nature of the object field region with its many SWS tips. This is unavoidable insofar as a structured Green function is desired: If it is introduced by a physical structure such as the metallic tips, here, it will lead to some degree of multiple scattering. Therefore, our next task is to assess what happens when two particles are present in the object field.

Let us thus consider the two-particles scenario illustrated in Fig. 4(b): Particle P1 does not move and remains in front of the third metallic tip from top, whereas particle P2 moves through the entire field of view. The observed output signal through the six field exits is plotted in Fig. 7, as was done for a single particle in Fig. 5. A central signal persists on tip \#4, which stems from the extra fixed particle, and the main features of Fig. 5 such as the two lobes at extreme P2 positions ( $\sim-80 \mathrm{~nm}$ and $+80 \mathrm{~nm}$ on tip \#2 and tip \#5, respectively) can also be identified. However, the field is more complex, and as expected, it is not the addition of two single particle fields.

To retrieve the information of the two particle positions, we have nevertheless attempted to make use of single-particle fields. If this is successful, it shall show that multiple scattering remains in acceptable proportion, and that single scattering, the basis of imaging, is the main operating mechanism of the dark-field hyperlens proposed here.

In order to estimate the respective positions of the two particles, we perform the scalar product between the output signal obtained with two particles, and the superposed fields of two single particles at any two positions. We drop the indices $j_{1}$ and $j_{2}$ for simplicity and denote the particle positions by indices 1 and 2:

$$
\vec{E}^{\text {superposed }}\left(y_{1}, y_{2}\right)=\vec{E}\left(y_{1}\right)+\vec{E}\left(y_{2}\right)
$$

If there were no multiple scattering, the two would be identical, and the notion of a point-spread-function (here a coherent one) could be somehow restored. But this is not the case. What we can hope, nevertheless, is that the field $\vec{E}^{\text {two,actual }}\left(y_{1}^{\prime}, y_{2}^{\prime}\right)$ most resembles $\vec{E}^{\text {superposed }}\left(y_{1}, y_{2}\right)$ for those

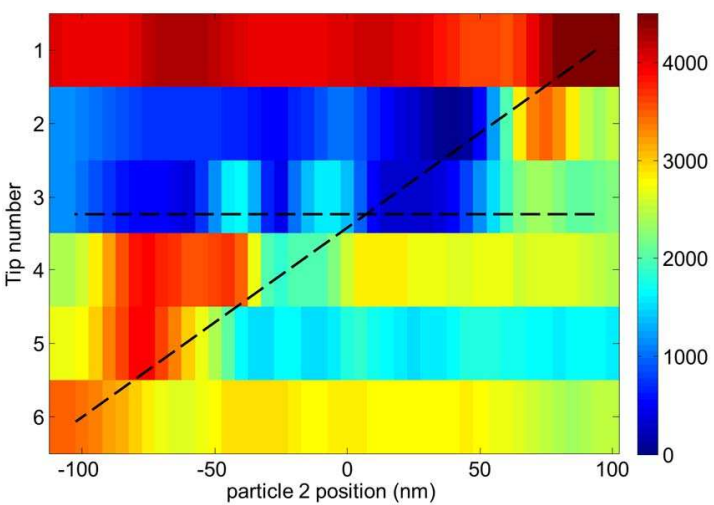

Fig. 7. (Color online) Hyperlens field modulus $|E|$ at the 6 exit tips at $\lambda_{\text {dark }}$, in a color map, for two Si particles, as a function of particle position. The dashed lines grossly represent the two expected "geometric" image positions, which may substantially differ from the signal peaks (see Fig. 5). position pairs $\left(y_{1}, y_{2}\right)$ which are as close as possible to the actual positions $\left(y_{1}^{\prime}, y_{2}^{\prime}\right)$. For our case with 43 different positions of $\mathrm{P} 2$, this means that, for each of them, we look in a set of $N^{2}=43^{2}=1849$ superposition cases which fits better in terms of the scalar product magnitude of the superposed field and the actual field:

$$
\begin{aligned}
B\left(y_{1}, y_{2}, y_{1}^{\prime}, y_{2}^{\prime}\right) & =\left|\vec{E}^{\text {superposed }}\left(y_{1}, y_{2}\right)^{+} \vec{E}^{\text {two,actual }}\left(y_{1}^{\prime}, y_{2}^{\prime}\right)\right| \\
& =\left|\left[\vec{E}\left(y_{1}\right)+\vec{E}\left(y_{2}\right)\right]^{+} \vec{E}^{\text {two,actual }}\left(y_{1}^{\prime}, y_{2}^{\prime}\right)\right|
\end{aligned}
$$

We thus calculated this quantity for the 43 simulated \{fixed P1 + moving P2\} situations of Figs. 4(b) and 7. Then, to get a "presence probability" for each of them, we sort among the $N^{2}$ results a reduced subset of the best ones, and attribute a "presence coefficient" at their associated $\left(y_{1}, y_{2}\right)_{\text {superposed }}$ positions. Such an estimator can be called the "intensity" or a pseudo-intensity, forming the pseudo-image of the two particles, discretized at the various positions $I_{j}$. We have thus taken the $N$ best results after looking at similar possibilities such as $N^{1 / 2}$ and $2 N$. $N$ out of $N^{2}$ is a reasonable guess to locate the expected particles with $1 / N$ relative accuracy, i.e., an accuracy of one position out of the $N$ pixelated ones. We then form for each of these $N$ guesses a contribution to $I_{j}$ at the two positions corresponding to the particle pair $\left(y_{1}, y_{2}\right)$. This is somewhat in the spirit of a histogram. We take, however, for these contributions a decreasing "presence coefficient" $C_{m}$, diminishing from $C_{1}=1$ for the best $B$ value ( $m=1$ ) to $C_{N} \sim 0.1$ for the $N$ th one. This use of $N$ out of the $N^{2}$ generated data retrieves a smoother distribution $I_{j}$ than the best guess alone (i.e., the best pair position), and we argue that it gives qualitatively a better account of the imaging capability of the device, notably in the presence of noise. For instance, by cumulating information from several secondranked $B$ values, we hope to more safely catch the case of particles at the actual position $\left(y_{1}^{\prime}, y_{2}^{\prime}\right)$, but whose signal is lowered due to coherent multiple scattering. As for the large amount of treatment that this retrieval seems to involve, it should be remarked that we are dealing with a correlation calculation that could be performed on the chip, much as coherent detection is practiced in coherent receivers for optical communications, and thus involving the same means that we invoked to make plausible the capability of mastering several phases and amplitudes on the tips of the illumination side of our proposed dark-field hyperlens.

The result of the procedure, a pseudo-intensity map $I_{j}$ for each of the 43 position pairs of our two-particles scenario, is given in Fig. 8(a). The fixed particle and the moving particle can easily be recognized. The detail of the $C_{m}$ series was found to be unimportant in terms of the allure of this pseudointensity map. One first delicate point, however, is the quite variable signal strength, which can be attributed to the variable amount of multiple scattering. In this first nonoptimized attempt, such fluctuations are important because no special precaution was taken to get more uniform response in the earlier design steps.

Next, one can wonder how severely the multiple scattering is blurring the picture itself. One way to look at this is to step back from our first philosophy of "full information exploitation" and to focus only on the first few best guesses to see how often the actual target is missed. To this end, Fig. 8(b) shows the four position pairs retrieved for the four best hits 
( $m=1$ to 4 ), with decreasing symbol size as a function of rank. It is seen that the information is essentially the same as that in Fig. 8(a), although Fig. 8(a) conveys a sense of accuracy that cannot be easily guessed from Fig. 8(b) alone.

The two main defective cases are pointed by arrows. They show positions where the moving particle is not seen, or in other words, the hyperlens is locally blind to it. We propose that these special situations which represent only a minority $(<10 \%)$ of the cases arise when the fixed particle scattering cancels most of the moving particle direct scattering, leaving only multiple scattering as the output field. This reasonable hypothesis should, however, be confirmed by a more refined analysis of the scattered fields in these cases, a work that we hope will complement this first report.

All these elements do not fundamentally jeopardize the dark-field hyperlens concept; they rather call for specific design directions along which they could be investigated and improved. Notably, along this line, the properties that have been recently pointed out in the canalization phenomena that allow a neat correspondence between an image outside a wire array and its well resolved image in the middle of the wire array could probably be exploited to get a much more pure imaging process than this first attempt. Our main message being the concept of the dark-field hyperlens itself, we hope that it can prompt further combinations with other clever concepts, so that it would lend itself more easily to a feasible realization.
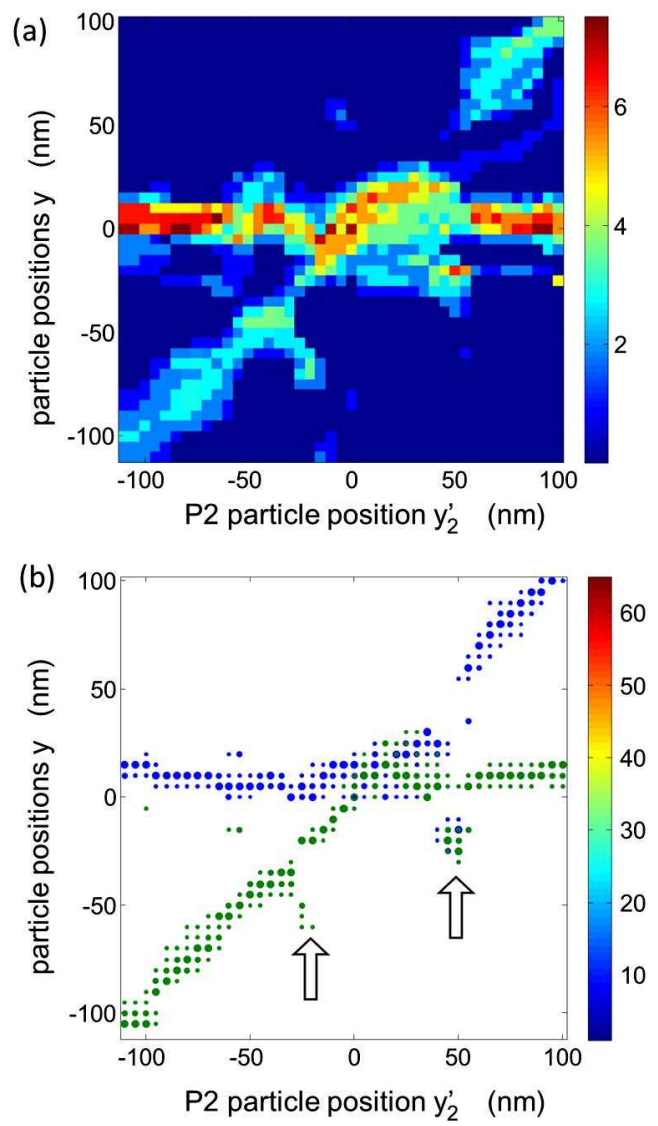

Fig. 8. (Color online) Particle position retrieval (a) estimator $I_{j}$ of the presence of two particles from the coherent projection onto two superposed one-particle "images." The fixed particle and the moving particle can easily be recognized. (b) Plot of positions associated

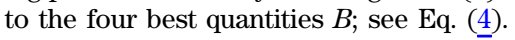

\section{CONCLUSION}

In conclusion, we have proposed a dark-field hyperlens scheme, able to transfer subwavelength scale information in parallel with a resolution $\sim 0.05 \times(\lambda / 2 n) \sim 20 \mathrm{~nm}$ from a field whose long dimension is $\sim 10$ times the resolution. This scheme is based on two facing fans of metallic tips inspired by the canalization phenomenon [5-10, 12-19], also termed endoscope [22]. To operate at near-infrared wavelength, the losses of these metallic tips can be partly obviated by transferring the information to silicon wires after $\mathrm{a} \sim 1 \mu \mathrm{m}$ propagation length, when the initial tips are separated by about $\lambda / 2 n_{\text {av }}$ with $n_{\text {av }}$ the average index of the array of silicon wires. The hyperlens input and output accesses are located on these silicon wires, and could consist of gratings for in- and out-coupling from air. Alternatively, the device could be placed next to a silicon photonic integrated circuit able to feed light to the hyperlens inputs with appropriate amplitudes and phases. The crucial point of obtaining information from small particles in transmission illumination mode is solved by means of our darkfield hyperlens scheme. This is the reason why we can get a signal without primary sources in the object field of our lens, but only with dielectric particles of moderate contrast, which has generally not been the case in earlier simulations and demonstrations. In our scheme, thanks to a proper excitation scheme of the input tips in phase and amplitude, a set of central exit tips produces a vanishing field in the absence of particles, exactly as occurs in classical dark-field microscopy with "ring" illumination.

We illustrated the operation of the hyperlens in a 2D simplified model, with a single particle of index $n_{\text {partic }}=2.8$ embedded in the $n=1.5$ background. The dark field area was composed of 4 out of 6 silicon exit tips, while the central metallic facing fans consist of seven tips separated by a $50 \mathrm{~nm}$ gap, making for a hyperlens field width of $220 \mathrm{~nm}$.

We then demonstrated that if two particles are present in the field, their positions can be acceptably retrieved thanks to a correlation analysis on the basis of the one-particle responses which generalizes the concept of point-spread-function. Such a generalization is needed because the subwavelength resolution requires the proximity of the tips to produce a "highly structured" Green function, a situation which unavoidably results in the occurrence of multiple scattering when more than one particle is involved, and fundamentally breaks down the usual version of the (coherent) point-spread-function concept. The concept can be generalized to expand the object field in the third dimension, and to discuss how to benefit from the capabilities from the integrated optics telecom studies to produce the required excitation distribution enabling these sensitive and novel dark-field systems.

\section{REFERENCES}

1. L. Novotny and B. Hecht, Principles of Nano-optics (Cambridge University, 2006).

2. J. B. Pendry, "Negative refraction makes a perfect lens," Phys. Rev. Lett. 85, 3966-3969 (2000).

3. N. Fang, H. Lee, C. Sun, and X. Zhang, "Sub-diffraction-limited optical imaging with a silver superlens," Science 308, 534-537 (2005).

4. Z. Liu, H. Lee, Y. Siong, C. Sun, and X. Zhang, "Far-field optical hyperlens magnifying sub-diffraction-limited objects," Science 315, 1686 (2007). 
5. M. G. Silveirinha, P. A. Belov, and C. R. Simovski, "Subwavelength imaging at infrared frequencies using an array of metallic nanorods," Phys. Rev. B 75, 035108 (2007).

6. M. G. Silveirinha, P. A. Belov, and C. R. Simosvski, "Ultimate limit of resolution of subwavelength imaging devices formed by metallic rods," Opt. Lett. 33, 1726-1728 (2008).

7. A. Rahman, S. Y. Kosulnikov, Y. Hao, C. Parini, and P. A. Belov, "Subwavelength optical imaging with an array of silver nanorods," J. Nanophoton. 5, 051601 (2011).

8. A. Rahman, P. A. Belov, M. G. Silveirinha, C. R. Simovski, Y. Hao, and C. Parini, "The importance of Fabry-Perot resonance and the role of shielding in subwavelength imaging performance of multiwire endoscopes," Appl. Phys. Lett. 94, 031104 (2009).

9. A. Rahman, P. A. Belov, Y. Hao, and C. Parini, "Periscope-like endoscope for transmission of a near field in the infrared range," Opt. Lett. 35, 142-144 (2010).

10. A. Rahman, P. A. Belov, and Y. Hao, "Tailoring silver nanorod arrays for subwavelength imaging of arbitrary coherent sources," Phys. Rev. B 82, 113408 (2010).

11. A. Ono, J. Kato, and S. Kawata, "Subwavelength optical imaging through a metallic nanorod array," Phys. Rev. Lett. 95, 267407 (2005).

12. P. Ikonen, C. Simovski, S. Tretyakov, P. Belov, and Y. Hao, "Magnification of subwavelength field distributions at microwave frequencies using a wire medium slab operating in the canalization regime," Appl. Phys. Lett. 91, 104102 (2007).

13. P. A. Belov, Y. Zhao, S. TSe, P. Ikonen, M. G. Silveirinha, C. R. Simosvski, S. Tretyakov, Y. Hao, and C. Parini, "Transmission of images with subwavelength resolution to distances of several wavelengths in the microwave range," Phys. Rev. B 77, 193108 (2008).

14. P. A. Belov, Y. Zhao, S. Sudhakaran, A. Alomainy, and Y. Hao, "Experimental study of the subwavelength imaging by a wire medium slab," Appl. Phys. Lett. 89, 262109 (2006).

15. P. A. Belov, C. R. Simovski, and P. Ikonen, "Canalization of subwavelength images by electromagnetic crystals," Phys. Rev. B 71, 193105 (2005).

16. P. A. Belov and M. G. Silveirinha, "Resolution of subwavelength transmission devices formed by a wire medium," Phys. Rev. E 73, 056607 (2006).

17. P. A. Belov, G. K. Palikaras, Y. Zhao, A. Rahman, C. R. Simovski, Y. Hao, and C. Parini, "Experimental demonstration of multiwire endoscopes capable of manipulating near-fields with subwavelength resolution," Appl. Phys. Lett. 97, 191905 (2010).

18. P. A. Belov, Y. Hao, and S. Sudhakaran, "Subwavelength microwave imaging using an array of parallel conducting wires as a lens," Phys. Rev. B 73, 033108 (2006).

19. P. A. Belov and Y. Hao, "Subwavelength imaging at optical frequencies using a transmission device formed by a periodic layered metal-dielectric structure operating in the canalization regime," Phys. Rev. B 73, 113110 (2006).

20. P. A. Belov, Y. Zhao, Y. Hao, and C. Parini, "Enhancement of evanescent spatial harmonics inside of materials with extreme optical anisotropy," Opt. Lett. 34, 527-529 (2009).

21. Y. Zhao, P. A. Belov, and Y. Hao, "Subwavelength internal imaging by means of a wire medium," J. Opt. A: Pure Appl. Opt. 11, 075101 (2009).

22. G. Shvets, S. Trendafilov, J. B. Pendry, and A. Sarychev, "Guiding, focusing, and sensing on the subwavelength scale using metallic wire arrays," Phys. Rev. Lett. 99, 053903 (2007).

23. J. Li, L. Fok, X. Yin, G. Bartal, and X. Zhang, "Experimental demonstration of an acoustic magnifying hyperlens," Nat. Mater. 8, 931-934 (2009).

24. M. I. Stockman, "Nanoplasmonics: past, present, and glimpse into future," Opt. Express 19, 22029-22106 (2011).

25. V. Z. Zayats, I. I. Smolyaninov, and A. A. Maradudin, "Nanooptics of surface plasmon polaritons," Phys. Rep. 408, 131-314 (2005).
26. A. M. H. Wong and G. V. Eleftheriades, "Advances in imaging beyond the diffraction limit," in Breakthroughs in Photonics 2011, IEEE Photon. J. 4, 561-656 (2012).

27. M. W. Davidson, "Darkfield Illumination" (National High Magnetic Field Laboratory, 2012), retrieved micro.magnet.fsu.edu/ primer/techniques/darkfield.html.

28. F. V. Ignatovitch and L. Novotny, "Real-time and backgroundfree detection of nanoscale particles," Phys. Rev. Lett. 96, 013901 (2006).

29. M. Brehm, T. Taubner, R. Hillenbrand, and F. Keilmann, "Infrared spectroscopic mapping of single nanoparticles and viruses at nanoscale resolution," Nano Lett. 6, 1307-1310 (2006)

30. F. Lemoult, M. Fink, and G. Lerosey, "Acoustic resonators for far-field control of sound on a subwavelength scale," Phys. Rev. Lett. 107, 064301 (2011).

31. G. Maire, F. Drsek, J. Girard, H. Giovannini, A. Talneau, D. Konan, K. Belkebir, P. C. Chaumet, and A. Sentenac, "Experimental demonstration of quantitative imaging beyond Abbe's limit with optical diffraction tomography," Phys. Rev. Lett 102, 213905 (2009).

32. H. Liu and K. J. Webb, "Resonance cones in cylindrically anisotropic metamaterials: a Green's function analysis," Opt. Lett. 36, 379-381 (2011).

33. H. Liu, Shivanand, and K. J. Webb, "Subwavelength imaging with nonmagnetic anisotropic bilayers," Opt. Lett. 34, 2243-2245 (2009).

34. L. Alekseyev, E. Narimanov, and J. Khurgin, "Super-resolution spatial frequency differentiation of nanoscale particles with a vibrating nanograting," Appl. Phys. Lett. 100, 011101 (2012).

35. F. Van Laere, T. Stomeo, C. Cambournac, M. Ayre, R. Brenot, H. Benisty, G. Roelkens, T. F. Krauss, D. Van Thourhout, and R. Baets, "Nanophotonic polarization diversity demultiplexer chip," J. Lightwave Technol. 27, 417-425 (2009).

36. D. Taillaert, F. Van Laere, M. Ayre, W. Bogaerts, D. van Tourhout, P. Bienstman, and R. Baets, "Grating couplers for coupling between optical fibers and nanophotonic waveguides,” Jpn. J. Appl. Phys. 45, 6071-6077 (2006).

37. T. Stomeo, F. Van Laere, M. Ayre, C. Cambournac, H. Benisty, D. Van Thourhout, R. Baets, and T. F. Krauss, "Integration of grating couplers with a compact photonic crystal demultiplexer on an InP membrane," Opt. Lett. 33, 884-886 (2008).

38. R. Halir, P. Cheben, S. Janz, D.-X. Xu, Í. Molina-Fernández, and J. G. Wangüemert-Pérez, "Waveguide grating coupler with subwavelength microstructures," Opt. Lett. 34, 1408-1410 (2009).

39. R. Halir, A. Ortega-Moñux, I. Molina-Fernandez, J. G. Wanguemert-Perez, P. Cheben, X. Dan-Xia, B. Lamontagne, and S. Janz, "Integrated optical six-port reflectometer in silicon on insulator," IEEE J. Lightw. Technol. 27, 5405-5409 (2009).

40. G. Roelkens, L. Liu, D. Liang, R. Jones, A. Fang, B. Koch, and J. Bowers, "III-V/silicon photonics for on-chip and intra-chip optical interconnects," Laser Photon. Rev. 4, 751-779 (2010).

41. R. Halir, G. Roelkens, A. Ortega-Monux, J. G. WanguemertPerez, and I. Molina-Fernandez, "High performance multimode interference couplers for coherent communications in silicon," Proc. SPIE 8007 80071B-80077 (2011).

42. Z. Han, A. Y. Elezzabi, and V. Van, "Experimental realization of subwavelength plasmonic slot waveguides on a silicon platform," Opt. Lett. 35, 502-504 (2010).

43. A. Klekamp and R. Münzner, "Calculation of imaging errors of AWG,” J. Lightwave Technol. 21, 1978-1986 (2003).

44. P. B. Johnson and R. W. Christy, "Optical constants of the noble metals," Phys. Rev. B 6, 4370-4379 (1972).

45. S. Huang, H. Wang, K.-H. Ding, and L. Tsang, "Subwavelength imaging enhancement through a three-dimensional plasmon superlens with rough surface," Opt. Lett. 37, 1295-1297 (2012).

46. H. Debrégeas, J. Decobert, N. Lagay, R. Guillamet, D. Carrara, O. Patard, C. Kazmierski, and R. Brenot, "Selective-area-growth technology for flexible active building blocks," in Integrated Photonics Research, Silicon and Nanophotonics, Technical Digest (CD) (Optical Society of America, 2012), p. IM2A.3. 